\title{
Cross-border outbreak of listeriosis caused by cold- smoked salmon, revealed by integrated surveillance and whole genome sequencing (WGS), Denmark and France, 2015 to 2017
}

Susanne Schjørring ${ }^{1}$, Sofie Gillesberg Lassen², Tenna Jensen ${ }^{3}$, Alexandra Moura ${ }^{4}$, Jette S Kjeldgaard ${ }^{5}$, Luise Müller ${ }^{2}$, Stine

Thielke ${ }^{3}$, Alexandre Leclercq ${ }^{4}$, Mylene M Maury ${ }^{4}$, Mathieu Tourdjman6, Marie-Pierre Donguy ${ }^{7}$, Marc Lecuit ${ }^{4,8}$, Steen Ethelberg ${ }^{2}$, Eva M Nielsen ${ }^{1}$

1. Department of Bacteria, Parasites and Fungi, Statens Serum Institut, Copenhagen, Denmark

2. Department of Infectious Disease Epidemiology and Prevention, Statens Serum Institut, Copenhagen, Denmark

3. The Danish Veterinary and Food Administration, Copenhagen, Denmark

4. Institut Pasteur, National Reference Centre and WHO collaborating Center for Listeria, Biology of Infection Unit, Paris, France

5. National Food Institute, Technical University of Denmark, Kgs. Lyngby, Denmark

6. Santé Publique France, the French Public Health Agency, Saint-Maurice, France

7. Ministry of Agriculture, and Forestry, Paris, France

8. Paris Descartes University, Sorbonne Paris Cité, Division of Infectious Diseases, Necker-Enfants Malades University Hospital, Institut Imagine, F-75743 Paris, France

\section{Correspondence: Susanne Schjørring (SSC@ssi.dk)}

Schiørring Susanne, Gillesberg Lassen Sofie, Jensen Tenna, Moura Alexandra, Kjeldgaard Jette S, Müller Luise, Thielke Stine, Leclercq Alexandre, Maury Mylene M, Tourdjman Mathieu, Donguy Marie-Pierre, Lecuit Marc, Ethelberg Steen, Nielsen Eva M. Cross-border outbreak of listeriosis caused by cold-smoked salmon, revealed by integrated surveillance and whole genome sequencing (WGS), Denmark and France, 2015 to 2017. Euro Surveill. 2017;22(50):pii=17-00762. https://doi. revealed by integrated surveillance and whole genor
org/10.2807/1560-7917.ES.2017.22.50.17-00762

Article submitted on 15 Nov 2017 / accepted on 14 Dec 2017 / published on 14 Dec 2017

In August 2017, an outbreak of six listeriosis cases in Denmark was traced to cold-smoked salmon, using epidemiological investigations and whole-genome sequencing (WGS) analyses. Exchange of genome sequences allowed identification in France of a food isolate from a salmon-derived product and a human isolate from 2016 within the same cgMLST cluster as the Danish isolates (L2-SL8-ST8-CT771). The salmon product came from a third European Union country. WGS can rapidly link human cases and food isolates across Europe.

\section{Identification of the outbreak}

In Denmark, on 23 August 2017, Statens Serum Institut (SSI) identified a genetic cluster of four human Listeria monocytogenes sequence type (ST) 8 isolates by core genome multilocus sequence typing (cgMLST) [1]. The allele calling was performed in BioNumerics (v7.6.2, Applied Maths, Belgium). We initiated an epidemiological investigation and notified the Danish Central Outbreak Management Group (collaboration between the Danish Veterinary and Food Administration (DVFA), the National Food Institute at the Technical University of Denmark (DTU) and SSI). On 25 August, two additional human isolates were found to belong to the same genetic cluster.
Raw sequence data of four outbreak isolates, SSI-AC382-5, are deposited at the European Nt Archive (ERS2039635-8).

\section{Case definition}

A confirmed case was defined as a person clinically diagnosed with listeriosis after 1 January 2017 with laboratory-confirmed L. monocytogenes ST8 clustering using cgMLST ( $\leq 5$ allelic distance, single linkage). Cases diagnosed before 1 January 2017 with an isolate belonging to this cluster were defined as probable cases.

\section{Case description and food exposure of Danish patients}

As of 25 August 2017, the genetic cluster comprised six cases; five confirmed and one probable. Laboratory sample dates ranged from 25 October 2015 to 21 August 2017 (Figure 1). The age of the cases ranged from 59 to 96 years (median 80 years) and four were women. All patients had underlying illness and no travel history. One patient died within 30 days of diagnosis. Epidemiological investigations including a standard questionnaire on exposures showed that all five confirmed cases had consumed cold-smoked and/ or cured salmon in the 30 days before disease onset. Four cases had bought the salmon in retail chain $X$. No other food-item was reported as consumed in high frequencies among cases. Epidemiological follow-up for 
Number of probable and confirmed cases of listeriosis, by month and year of laboratory sample date, sex and country, Denmark and France, 2015-2017 ( $\mathrm{n}=7$ cases)

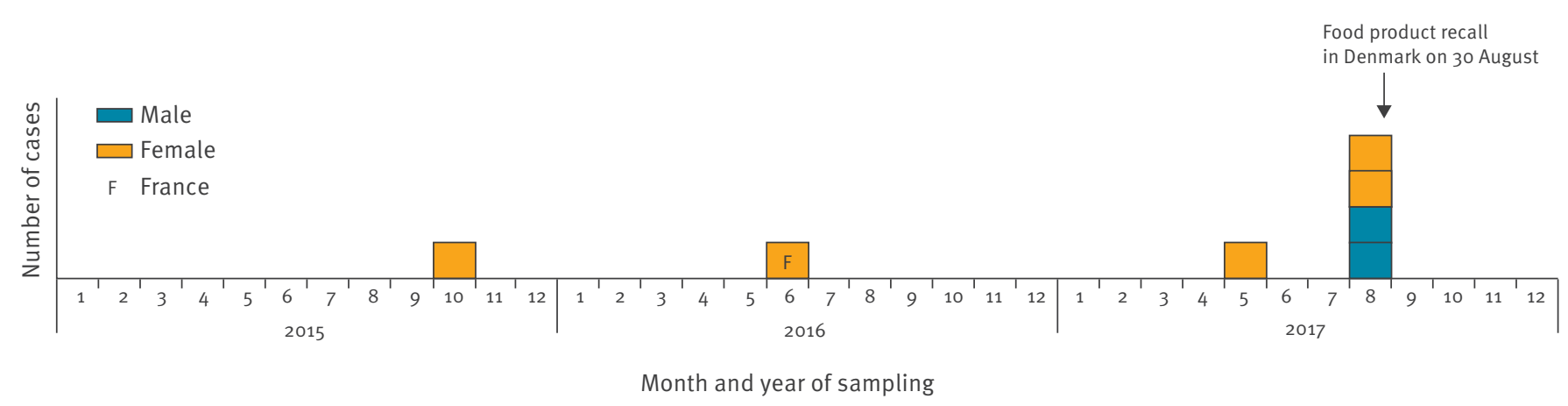

Unless otherwise specified with F, cases are from Denmark.

the probable case did not include information on fish consumption.

\section{Food investigation and control measures}

On 29 August 2017, a comparison between the human outbreak isolates and 16 L. monocytogenes ST8 foodand environmental isolates identified in Denmark from 2014 to August 2017 showed that the human isolates clustered with a food isolate from cold-smoked salmon, cut and packaged at company $Y$ in Poland (zero to two allelic differences using cgMLST). $L$. monocytogenes had been detected on 31 July 2017 at levels of $110 \mathrm{CFU} / \mathrm{g}$ (threshold: $100 \mathrm{CFU} / \mathrm{g}$ ) at the end of shelf life. The product was widely sold in Denmark and had been sampled by the DVFA in retail chain $X$, as part of a consumer exposure survey (i.e. analyses project on retail packages). Because the L. monocytogenes concentration had been just above the accepted limit and found at the end of the product shelf life a recall of this batch was not conducted. However, due to the positive finding, follow-up sampling had been performed on the 9 and 10 August 2017 from the central storage unit of retail chain X. L. monocytogenes had been isolated from two batches analysed before end of shelf life. In one sample from the same batches, which was also analysed at the end of the shelf life, on 28 August 2017 a L. monocytogenes level of $240 \mathrm{CFU} / \mathrm{g}$ was found. Isolates from the follow-up samples had zero to four allelic differences to the human outbreak isolates using cgMLST.

The human outbreak sequences were also compared to all L. monocytogenes ST8 genomes derived from clinical samples in Denmark from 2012 onwards. Although ST8 genomes from Danish patients in the period 2012-2017 showed high diversity, the outbreak isolates clearly formed a distinct cgMLST cluster with 16 allelic differences to the nearest isolates outside the genetic outbreak cluster and a maximum of nine allelic differences within the cluster (Figure 2a). We investigated the relatedness of outbreak isolates further by single-nucleotide polymorphisms (SNP) analysis performed by both SSI and DTU using two analysis pipelines: Northern Arizona SNP Pipeline (NASP) [2] and CSI Phylogeny version 1.4 from Center for Genomics Epidemiology (CGE), DTU [3] leading to the same conclusion. The SNP analysis showed a maximum of nine SNPs between any two isolates in the cluster (Figure $2 b$ ). The food isolate sampled in Denmark in July was identical by SNP analysis to one of the Danish patient outbreak isolates from August 2017.

On 30 August 2017, DVFA advised retail chain $X$ to recall all cold-smoked salmon produced at company $Y$. This advice was based on the elevated number of $L$. monocytogenes (240 CFU/g) found in the product at the end of shelf-life and the link to the outbreak. Retail chain $X$ voluntarily recalled both cold-smoked and cured salmon produced at company $Y$. As part of the recall procedure, retail chain $X$ informed company $Y$ on the situation. Information from company $Y$, provided by the Polish food authorities via the European Union Rapid Alert System for Food and Feed (RASFF), showed that the implicated batches were exclusively sold via retail chain $\mathrm{X}$ and only in Denmark.

\section{International enquiry and investigation in France}

On 31 August 2017, the outbreak was notified internationally on different communication platforms: (i) the European Epidemic Intelligence Information System for food- and waterborne diseases (EPIS-FWD) reference UI-426, (ii) Early Warning and Response System (EWRS) notification reference EWRS20170831DKoo01 and (iii) RASFF notification reference RASFF-2017.1319. The EPIS-FWD platform allowed sharing of files with assembled genomic sequence data. SSI distributed raw sequence data of four outbreak isolates (SSI$A C 382-A C 385)$ on a local ftp server at SSI.

The French National Reference Centre (NRC) for Listeria (Institut Pasteur, Paris), compared the 


\section{FIGURE 2}

Whole genome sequencing based typing of Listeria monocytogenes ST8 isolates as part of of a cross-border listeriosis outbreak investigation, Denmark and France, 2015-2017

A. Minimum spanning tree of cgMLST allelic profiles ${ }^{\mathrm{a}}$

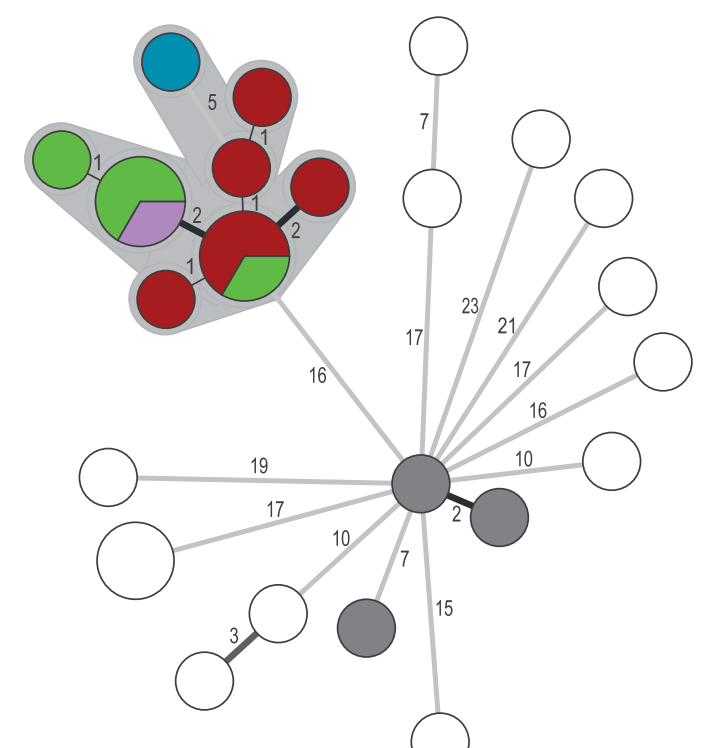

B. Maximum parsimony tree based on whole-genome sequencing (WGS) data ${ }^{b}$

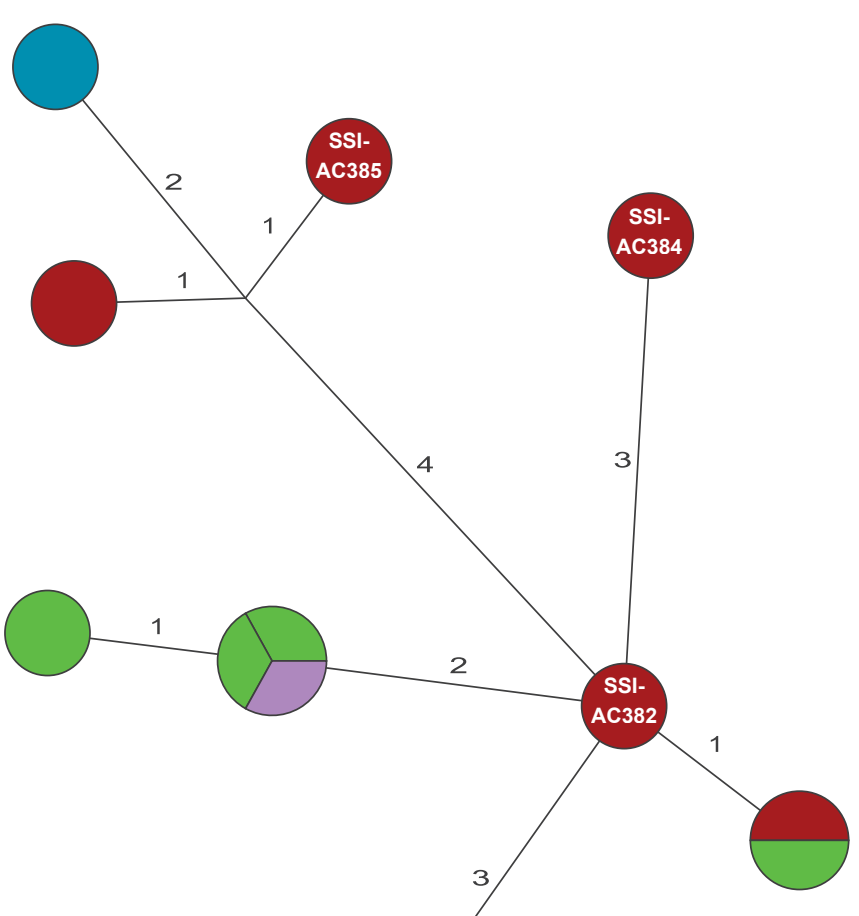

Human Denmark

$\square$ Food Denmark

$\square$ Human France

$\square$ Food France

$\square$ Poultry processing facilities Norway

$\square$ Salmon processing facilities Norway

$\square$ Human Denmark, unrelated to the outbreak

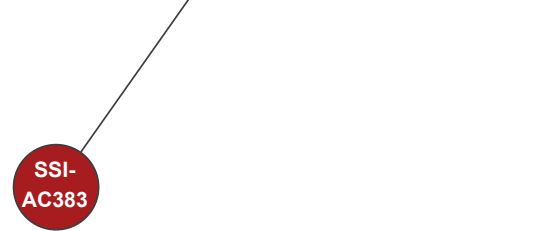

17

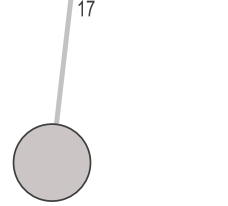

cgMLST: core genome multilocus sequence typing; SSI: Statens Serum Institut.

The size of nodes corresponds to the number of isolates. Branch lengths represent the SNP difference.

a Minimum spanning tree of cgMLST allelic profiles of $21 \mathrm{~L}$. monocytogenes ST8 isolates from Danish patients in the period $2012-2017$, 1 isolate from a probable French case 2016 and food isolates sampled in Denmark (4 isolates; July-August 2017) and France (1 isolate; September 2017) from products produced by company Y. The outbreak cluster is shaded in grey. Published sequences of ST8 isolates (2001-2013) from a study [17] on persistence in a poultry processing facility (SRR3099221, SRR3099222) and a salmon processing facility (SRR3099223, SRR3099224, SRR3099225) were included.

${ }^{b}$ Maximum parsimony tree based on whole-genome sequencing (WGS) data showing single-nucleotide polymorphisms (SNPs) within the L. monocytogenes ST8 isolates from patients and food sources in the genetic outbreak cluster. One isolate (SSI-AC382, ERS2039635) from the cluster was used as the reference when using NASP. 
sequences of the Danish human isolates against its database, using cgMLST as previously described $[1,4]$. A human isolate from a French resident belonged to the same cluster (L2-SL8-ST8-CT771) as the Danish isolates. This French probable case, a female patient in her mid-80s, was diagnosed in June 2016. Epidemiological investigations carried out by Santé Publique France were inconclusive, since food consumption history was not available at the time of diagnosis nor could information on travel to Denmark be retrieved, as the person had since died.

On 6 September 2017, an official control by the Ministry of Economy was carried out at a French retailer where a kosher chilled cured salmon was sampled for analysis. The sample was contaminated with L. monocytogenes at the level of $460 \mathrm{CFU} / \mathrm{g}$ and the salmon producer was company $Y$. An isolate was sent to the French NRC for typing and showed to belong to the same cgMLST type as the Danish outbreak (Figure 2). Further investigations on the food product confirmed that it had not been further processed after production in Poland. The product was recalled and no human cases were linked to its consumption as of beginning of December 2017.

The other nine countries that replied to the EPISFWD UI-426 notification (Austria, Finland, Germany, Luxembourg, the Netherlands, Norway, Slovenia, Sweden, United Kingdom) did not report any human or food isolates linked to the Danish outbreak. However, after submission of this report, at the end of November, we were informed through EPIS about three genetically linked human isolates in Germany.

\section{Discussion}

Here we report on a listeriosis outbreak and highlight the value of rapidly comparing the genomes of human and food/environmental isolates at the national and international levels.

The fact that the contaminated salmon products identified in Denmark and France were from different batches suggests environmental contamination possibly at the production facility at company $Y$. It is too early to assess whether any measures taken at company $Y$ have been effective in controlling the outbreak. However, experiences from previous investigations suggest that once $L$. monocytogenes is detected in one product, the whole production site should be subject to a thorough inspection, and sampling with special attention to all the possible contamination/cross contamination issues before implementing corrective measures $[5,6]$. Moreover, the risk for $L$. monocytogenes persistent strains in the production environment requires the close monitoring for several years to ensure the elimination of these $[7,8]$.

Since WGS was introduced for routine surveillance in Denmark, a number of listeriosis outbreaks have been detected and solved, including outbreaks involving cold-smoked ready-to-eat sliced fish products [5]. The present investigation further reinforces the suspicion that ready-to-eat fish products are important sources of $L$. monocytogenes infections in Denmark, as well as in other countries.

Though only involving a low number of isolates, WGS $L$. monocytogenes surveillance and communication between countries allowed us to detect and rapidly solve this salmon-associated outbreak, leading to food product recall in two European countries. Compared with previous typing methods, WGS has a higher discriminatory power and the ability to determine genetic distance between isolates. The introduction of WGS for surveillance of food-borne infections has shown that it improves outbreak detection and facilitates outbreak investigations and likely helps reduce the number of infections [4,9-16]. The EPIS-FWD communication platforms allowed for the communication to link cases across borders. However, currently cross-border outbreaks are only detected when case numbers in at least one country exceed normal levels and are notified internationally. Therefore, a possible future system for easy exchange of and comparison of WGS data, e.g. by the use of an agreed cgMLST nomenclature, across borders will enable the identification of more dispersed outbreaks as well as cross-border links between food samples and human infections. This report highlights that by the application of cross-disciplinary and real-time cross-border comparison of WGS data, L. monocytogenes infections can be prevented and thereby providing safer food for at-risk groups such as the elderly, immunodeficient individuals and pregnant women.

\section{Acknowledgements}

We thank the Danish Clinical Microbiological Laboratories for rapid referral of isolates and support of the surveillance system. In addition, we thank the physicians and other health personnel for supplying clinical information; Marie Walther, Charlotte Munkstrup, Louise La Barbera Kastberg, for their assistance in interviewing patients. Furthermore, we thank Christian Vråby Pedersen and Anette Holm (SSI), Anne Mette Seyfarth (DFVA) the French National reference center technicians, and the PIBnet platform (Institut Pasteur) for excellent technical expertise in performing whole-genome sequencing. We also thank the staff at the DFVA Laboratory in Ringsted, Denmark and the regional food inspection units for collecting the environment and product samples. Part of this work was supported by the European Union's Horizon 2020 research and innovation program through COMPARE under grant agreement No. 643476.

\section{Conflict of interest}

None declared.

Authors' contributions

SS and SGL: participated in the outbreak investigation and drafted the manuscript. SS: conducted the typing of human isolates and comparison of human isolates as well as 
comparison between human, environmental and product isolates. SGL: contributed to the clinical follow-up, patient interviews and the epidemiological investigation. TJ: was in charge of the trace-back investigations. AM, MMM, AL, ML: conducted the typing of human and food isolates in France and contributed to the writing of the manuscript. JSK: compared human, environmental and product isolates. LM: contributed to the clinical follow-up and the epidemiological investigations. ST: was involved in the trace-back investigations. AL: conducted the typing of human isolates and comparison of human isolates as well as comparison between human, environmental and product isolates in France and participated in the writing of the manuscript. MT, MD: conducted the human and food epidemiological investigations in France. SE: contributed to the epidemiological investigations and contributed to the writing of the manuscript. EMN: contributed to the laboratory and typing investigations as well as contributed to the writing of the manuscript.

\section{References}

1. Moura A, Criscuolo A, Pouseele H, Maury MM, Leclercq A, Tarr C, et al. Whole genome-based population biology and epidemiological surveillance of Listeria monocytogenes. Nat Microbiol. 2016;2:16185. https://doi.org/10.1038/ nmicrobiol.2016.185 PMID: 27723724

2. Sahl JW, Lemmer D, Travis J, Schupp JM, Gillece JD, Aziz M, et al. NASP: an accurate, rapid method for the identification of SNPs in WGS datasets that supports flexible input and output formats. Microb Genom. 2016;2(8):e000074. https://doi. org/10.1099/mgen.0.000074 PMID: 28348869

3. Kaas RS, Leekitcharoenphon P, Aarestrup FM, Lund O. Solving the problem of comparing whole bacterial genomes across different sequencing platforms. PLoS One. 2014;9(8):e104984. https://doi.org/10.1371/journal.pone.0104984 PMID: 25110940

4. Moura A, Tourdjman M, Leclercq A, Hamelin E, Laurent E, Fredriksen N, et al. Real-Time Whole-Genome Sequencing for Surveillance of Listeria monocytogenes, France. Emerg Infect Dis. 2017;23(9):1462-70. https://doi.org/10.3201/ eid2309.170336 PMID: 28643628

5. Kvistholm Jensen A, Nielsen EM, Björkman JT, Jensen T, Müller L, Persson S, et al. Whole-genome Sequencing Used to Investigate a Nationwide Outbreak of Listeriosis Caused by Ready-to-eat Delicatessen Meat, Denmark, 2014. Clin Infect Dis. 2016;63(1):64-70. https://doi.org/10.1093/cid/ciw192 PMID: 27025820

6. Gillesberg Lassen S, Ethelberg S, Björkman JT, Jensen T, Sørensen G, Kvistholm Jensen A, et al. Two listeria outbreaks caused by smoked fish consumption-using whole-genome sequencing for outbreak investigations. Clin Microbiol Infect. 2016;22(7):620-4. https://doi.org/10.1016/j.cmi.2016.04.017 PMID: 27145209

7. Holch A, Webb K, Lukjancenko O, Ussery D, Rosenthal BM, Gram L. Genome sequencing identifies two nearly unchanged strains of persistent Listeria monocytogenes isolated at two different fish processing plants sampled 6 years apart. Appl Environ Microbiol. 2013;79(9):2944-51. https://doi. org/10.1128/AEM.03715-12 PMID: 23435887

8. Ferreira V, Wiedmann M, Teixeira P, Stasiewicz MJ. Listeria monocytogenes persistence in food-associated environments: epidemiology, strain characteristics, and implications for public health. J Food Prot. 2014:77(1):150-70. https://doi. org/10.4315/0362-028X.JFP-13-150 PMID: 24406014

9. Yde M, Naranjo M, Mattheus W, Stragier P, Pochet B, Beulens $\mathrm{K}$, et al. Usefulness of the European Epidemic Intelligence Information System in the management of an outbreak of listeriosis, Belgium, 2011. Euro Surveill. 2012;17(38):20279. PMID: 23040965

10. Ronholm J, Nasheri N, Petronella N, Pagotto F. Navigating Microbiological food safety in the Era of Whole-Genome sequencing. Clin Microbiol Rev. 2016;29(4):837-57. https://doi. org/10.1128/CMR.00056-16 PMID: 27559074

11. Kwong JC, Mercoulia K, Tomita T, Easton M, Li HY, Bulach DM, et al. Prospective whole-genome sequencing enhances national surveillance of Listeria monocytogenes. J Clin Microbiol. 2016;54(2):333-42. https://doi.org/10.1128/ JCM.02344-15 PMID: 26607978

12. Jackson BR, Tarr C, Strain E, Jackson KA, Conrad A, Carleton $\mathrm{H}$, et al. Implementation of Nationwide Real-time Whole-genome Sequencing to Enhance Listeriosis Outbreak Detection and Investigation. Clin Infect Dis. 2016;63(3):380-6. https://doi. org/10.1093/cid/ciw242 PMID: 27090985
13. Kleta S, Hammerl JA, Dieckmann R, Malorny B, Borowiak M, Halbedel S, et al. Molecular Tracing to Find Source of Protracted Invasive Listeriosis Outbreak, Southern Germany, 2012-2016. Emerg Infect Dis. 2017;23(10):1680-3. https://doi. org/10.3201/eid2310.161623 PMID: 28930013

14. Comandatore F, Corbella M, Andreoli G, Scaltriti E, Aguzzi M, Gaiarsa S, et al. Genomic Characterization Helps Dissecting an Outbreak of Listeriosis in Northern Italy. PLoS Curr. 2017;9:ecurrents.outbreaks.633fd8994egfo6f31b3494567c7e5 04C680c. PMID: 28856063

15. Dahl V, Sundqvist L, Hedenström I, Löfdahl M, Alm E, Ringberg $\mathrm{H}$, et al. A nationwide outbreak of listeriosis associated with cold-cuts, Sweden 2013-2014. Infect Ecol Epidemiol. 2017;7(1):1324232. https://doi.org/10.1080/20008686.2017.13 24232 PMID: 28649304

16. Schmid D, Allerberger F, Huhulescu S, Pietzka A, Amar C, Kleta $\mathrm{S}$, et al. Whole genome sequencing as a tool to investigate a cluster of seven cases of listeriosis in Austria and Germany, 2011-2013. Clin Microbiol Infect. 2014;20(5):431-6. https://doi. org/10.1111/1469-0691.12638 PMID: 24698214

17. Fagerlund A, Langsrud S, Schirmer BC, Møretrø T, Heir E. Genome Analysis of Listeria monocytogenes Sequence Type 8 Strains Persisting in Salmon and Poultry Processing Environments and Comparison with Related Strains. PLoS One. 2016;11(3):e0151117. https://doi.org/10.1371/journal. pone.0151117 PMID: 26953695

\section{License and copyright}

This is an open-access article distributed under the terms of the Creative Commons Attribution (CC BY 4.0) Licence. You may share and adapt the material, but must give appropriate credit to the source, provide a link to the licence, and indicate if changes were made.

This article is copyright of the authors, 2017. 